\title{
Rapid tests for detection of carbapenemase producers in $P$. aeruginosa; what do we really need?
}

\section{Pruebas rápidas para la detección de Pseudomonas aeruginosa productora de carbapenemasas. ¿Qué necesitamos realmente?}

\author{
Laurent Poirel ${ }^{\mathrm{a}, \mathrm{b}, \mathrm{c}, *}$, Patrice Nordmann ${ }^{\mathrm{a}, \mathrm{b}, \mathrm{c}, \mathrm{d}}$ \\ a INSERM U914 "Emerging Resistance to Antibiotics", France \\ ${ }^{\mathrm{b}}$ Medical and Molecular Microbiology, Department of Medicine, Faculty of Science, University of Fribourg, Switzerland \\ ${ }^{c}$ Centre National Associé - Centre de Référence des Résistances aux Antibiotiques, Le Kremlin-Bicêtre, France \\ ${ }^{\mathrm{d}}$ Hopital Fribourgeois-hôpital Cantonal, Fribourg, Switzerland
}

Resistance to carbapenems in Pseudomonas aeruginosa is a major issue since that species is intrinsically resistant to many antibiotics and therefore, as soon as some acquired resistance traits occur, carbapenems become rapidly the ultimate therapeutic options. ${ }^{1}$ Carbapenem resistance in $P$. aeruginosa may occur through the loss of an outer-membrane protein named OprD that results from mutations or truncations in the corresponding gene. ${ }^{2}$ In Europe, that mechanism accounts for $15-20 \%$ of the imipenemresistant $P$. aeruginosa isolates. It is not transmissible since it is chromosomally-encoded and interferes with imipenem susceptibility only, meropenem and doripenem as well as broad-spectrum cephalosporins remaining effective against OprD-deficient strains (if no additional acquired mechanism). However, the acquisition of carbapenemase genes is another mechanism of carbapenem resistance in $P$. aeruginosa that is increasingly identified wordwide. ${ }^{3}$ That resistance trait is more worrisome since it corresponds to genes acquired through horizontal gene transfers that may disseminate, and impacts all carbapenem molecules that are all hydrolyzed by those enzymes. In $P$. aeruginosa, carbapenemases are mainly metallo- $\beta$-lactamases (MBLs) that hydrolyze all carbapenems very efficiently. ${ }^{4}$ They are not inhibited by $\beta$ lactamase inhibitors such as clavulanic acid and tazobactam and also confer resistance to other $\beta$-lactams such as broad-spectrum cephalosporins.

Therefore, an accurate and rapid detection of carbapenemase production in $P$. aeruginosa is extremely important. Such rapid detection may contribute to facilitate the antibiotic stewardship and treatment of infected patients and to prevent the further spread of those carbapenem-resistant isolates.

Therefore, in order to optimize infection control and antimicrobial therapy, advances in detection methods are crucial. Use

\footnotetext{
* Corresponding author.

E-mail address: laurent.poirel@unifr.ch (L. Poirel).
}

of molecular biology and in particular PCR-based techniques is being the key-approach for rapid and accurate identification of those resistance traits. The main advantages of molecular techniques are sensitivity, specificity, and the relative rapidity of these methods. Without molecular techniques in routine laboratories, identification of resistance mechanisms is often fastidious and time-consuming.

However, there are some drawbacks with those molecular techniques, the main ones are: (i) they are expensive, (ii) they do require some significant expertise, (iii) they do not fully replace the antibiogram since they only target some specific molecular determinants but do not evaluate the real susceptibility of the isolate to a large variety of drugs, (iv) the correlation between gene identification and resistance is not always observed (gene expression and modulation), and (v) by definition they do not identify the novel and emerging resistance genes.

That is the reason why biochemical and phenotypical methods have still to be considered as interesting tools. In the study performed by Lucena et al. in this issue,,$^{5}$ phenotypic tests for detection of MBL production have been compared with an impressive collection of metallo- $\beta$-lactamase producers, mostly of the SPM type. The double-disk synergy test (DDST), the Combined-Disk (CD) assay, and the MBLE-test have been compared. They all show some advantages and disadvantages, but one main shortcoming is the lack of clear cut-off to accurately interpret the obtained results. Depending on the strain background (additional resistance mechanisms or not), and on the user's technical skills, variable results and interpretations may be observed.

We believe that those diagnostic techniques, in order to be used, must not only be affordable and rapid, but overall must be easy to perform and interpret. Routine laboratories cannot rely on complicated screening strategies that, in addition, may provide difficult-to-interpret results. Efforts must be placed in developing those tests that may be used in any clinical laboratory, and not only in those with advanced technologies or highly qualified personal. 
One recently developed technique aiming to rapidly identify carbapenemase producers, either in Pseudomonas spp. and in Enterobacteriaceae, is the Carba NP test that corresponds to an efficient and reliable diagnostic tool for identification of those enzymes. ${ }^{6}$ The main advantages of this test are that it is extremely rapid and costless, by contrast to most of the other known techniques. It actually represents a breakthrough for detection of carbapenemase producers with results obtained in less than two hours, either directly from colonies but also from clinical samples such as blood cultures and urines. ${ }^{7,8}$ Another major advantage of this test is that it can be implemented in any clinical laboratories due to its simplicity. It is therefore well adapted to the current needs, i.e. improving the hygiene control and the antimicrobial therapy. In a country such as Brazil where the study from Lucena et al. has been performed, ${ }^{5}$ it might really provide an added-value since not only its low price and low-expertise requirement will surely fit to the expectations, but also the current and very worrisome epidemiology (with huge dissemination of SPM-1-producing $P$. aeruginosa strains all over the country ${ }^{9,10}$ ) will make it an essential tool.

Public health authorities around the world prompt to establish guidelines recommending strict and accurate monitoring of antimicrobial resistance should facilitate the development and dissemination of those kinds of methods that correspond to the needs in the "real" daily life.

\section{References}

1. Nordmann P, Naas T, Fortineau N, Poirel L. Superbugs in the coming new decade; multidrug resistance and prospects for treatment of Staphylococcus aureus, Enterococcus spp. and Pseudomonas aeruginosa in 2010. Curr Opin Microbiol. 2007; 10:436-40

2. Nordmann P. Gram-negative bacteria with resistance to carbapenems. Med Sci (Paris). 2010;26:950-9.

3. Poirel L, Pitout JD, Nordmann P. Carbapenemases: molecular diversity and clinical consequences. Future Microbiol. 2007;2:501-12.

4. Walsh TR, Toleman MA, Poirel L, Nordmann P. Metallo- $\beta$-lactamases: the quiet before the storm? Clin Microbiol Rev. 2005;18:306-25.

5. Lucena A, Dalla Costa LM, Da Silva Nogueira K, Matos AP, Gales AC, Raboni $\mathrm{SM}$. Comparison of phenotypic tests for the detection of metallo- $\beta$-lactamases in clinical isolates of Pseudomonas aeruginosa. Enferm Infect Microbiol Clin. 2014;32:625-30.

6. Nordmann P, Poirel L, Dortet L. Rapid detection of carbapenemase-producing Enterobacteriaceae. Emerg Infect Dis. 2012;18:1503-7.

7. Dortet L, Bréchard L, Cuzon G, Poirel L, Nordmann P. Strategy for rapid detection of carbapenemase-producing Enterobacteriaceae. Antimicrob Agents Chemother. 2014;58:2441-5.

8. Dortet L, Bréchard L, Poirel L, Nordmann P. Rapid detection of carbapenemaseproducing Enterobacteriaceae from blood cultures. Clin Microbiol Infect. 2014;20:340-4

9. Gales AC, Menezes LC, Silbert S, Sader HS. Dissemination in distinct Brazilian regions of an epidemic carbapenem-resistant Pseudomonas aeruginosa producing SPM metallo- $\beta$-lactamase. J Antimicrob Chemother. 2003;52: 699-702.

10. Poirel L, Magalhaes M, Lopes M, Nordmann P. Molecular analysis of metallo- $\beta$-lactamase gene bla $a_{\mathrm{SPM}-1}$-surrounding sequences from disseminated Pseudomonas aeruginosa isolates in Recife, Brazil. Antimicrob Agents Chemother. 2004;48:1406-9. 